\title{
Article
}

\section{Three new species of Agaricus section Minores from China}

\author{
He MQ ${ }^{1,2,3}$, Hyde $\mathrm{KD}^{3}$, Wei $\mathrm{SL}^{4}$, Xi YL ${ }^{4}$, Cheewangkoon $\mathrm{R}^{* 1}$ and Zhao RL*2,5 \\ ${ }^{1}$ Department of Entomology and Plant Pathology, Faculty of Agriculture, Chiang Mai University, Chiang Mai, 50200, \\ Thailand. \\ ${ }^{2}$ State key laboratory of Mycology, Institute of Microbiology, Chinese Academy of Sciences, Beijing, 100101, China. \\ ${ }^{3}$ Center of Excellence in Fungal Research, Mae Fah Luang University, Chiang Rai, 57100, Thailand. \\ ${ }^{4}$ Gansu Engineering Laboratory of Applied Mycology, Hexi University, 846th Huancheng North Road, Ganzhou District, \\ Zhangye 734000, Gansu, China. \\ ${ }^{5}$ College of Life Sciences, University of Chinese Academy of Sciences, Huairou District, Beijing, 100408, China.
}

He MQ, Hyde KD, Wei SL, Xi YL, Cheewangkoon R, Zhao RL 2018 - Three new species of Agaricus section Minores from China. Mycosphere 9(2), 189-201, Doi 10.5943/mycosphere/9/2/3

\begin{abstract}
Agaricus subgenus Minores section Minores has the richest species in the genus Agaricus and worldwide distribution. In this paper, the phylogenetic trees generated by Maximum Likelihood and Bayesian analyses for section Minores were conducted using ITS, LSU and tef1- $\alpha$ genes sequences from 105 species. The molecular phylogenetic analysis showed there were three new lineages from Chinese specimens within this section, and the following morphological examination supported them as three new species. We named them as A. parvibrunneus, A. pseudominipurpureus and A. yanzhiensis respectively. Descriptions, colour photographs and illustrations of those new species are presented in detail.
\end{abstract}

Key words - Agaricaceae - Phylogeny - Taxonomy

\section{Introduction}

Agaricus L. 1753 (Agaricaceae, Agaricales) is a well-studied genus with many species that are famous because of their high commercial value, such as A. bisporus (J.E. Lange) Imbach and $A$. subrufescens Peck.. Regional monographs for Agaricus have been published in recent years, such as monographs from Europe (Parra 2008, 2013) and Northern America (Kerrigan 2016). These monographs have given some detailed introductions among Agaricus and also well documented Agaricus species from those areas (Parra 2008, 2013, Kerrigan 2016). Agaricus is a genus with great species diversity, and today more than 500 species are recognized (Zhao et al. 2011, 2016, Chen et al. 2017, He et al. 2017).

Systematics of Agaricus drew a great attention of mycologists in the past ten years especially when the molecular data became available. More recently, the comprehensive infrageneric studies segregated this genus into six subgenera and 21 sections (Zhao et al. 2016, Chen et al. 2017). Agaricus section Minores was raised to subgenus according to its phylogenetic position and divergence time (Zhao et al. 2016). Section Minores is the section having the richest species in this genus, and 27 Asian species were introduced from Greater Mekong Subregion and southern China (Chen et al. 2017), latter the monograph of section Minores of China reported 19 species (He et al. 2017). Now this section contains about 80 named species and is expected to harbor at least 200 species within this section in the world (Chen et al. 2017, He et al. 2017). 
The morphological characteristics of A. sect. Minores generally are as follows: basidiomes slender, small or medium sized; annulus simple, thin; odour of almonds or anise; basidiomes turning yellow on touching; the $\mathrm{KOH}$ and Schäffer's reactions positive. However, in general, the morphological characteristics used for species distinction are scarce, especially between closely related species. Then in such case, the molecular data becomes essential for an unequivocal identification. Such as for the three species A. armandomyces M.Q. He \& R.L. Zhao, A. kerriganii L.A. Parra, B. Rodr., A. Caball., M. Martín-Calvo \& Callac and A. edmondoi L.A. Parra, Cappelli \& Callac, which cannot be distinguished easily morphologically but require molecular data (He et al. 2017).

During our study of Agaricus in China, some new Minores members have been introduced in the previous studies, such as A. gemloides M.Q. He \& R.L. Zhao, A. coccyginus M.Q. He \& R.L. Zhao, A. globosporus M.Q. He \& R.L. Zhao and so on (He \& Zhao 2015, Li et al. 2016, He et al. 2017). In this study, based on morphological characteristics and multi-locus phylogenetic analyses, we introduce three more new species here from China.

\section{Materials \& Methods}

\section{Morphologic study}

Specimens were collected during rainy seasons (July to September) from China, photographs of fresh specimens were taken immediately in situ, basidiomes were wrapped in aluminium foil or put in plastic boxes separately. Morphological characteristics including odour, basidiome size, colour and chemical reaction were recorded when the fruiting body are fresh. Every specimen was dried in an electrical food drier at $65{ }^{\circ} \mathrm{C}$ until no more moisture left, kept in a plastic ziplock bag and deposited in Herbarium Mycologicum Academiae Sinicae (HMAS). Anatomical and cytological characteristics including basidiospores, basidia, cystidia and pileipellis were observed under an Olympus CX31 microscope. At least 20 measurements were made. Data were analyzed and recorded as $X=$ the mean of length by width $\pm \mathrm{SD}, \mathrm{Q}=$ the quotient of basidiospore length to width, and $\mathrm{Q}_{\mathrm{m}}=$ the mean of $\mathrm{Q}$ values \pm SD. All the protocols of morphological study followed Largent's methodology (Largent 1986). Macrochemical reactions including KOH reaction and Schäffer's reaction are followed Chen et al. (2015).

\section{DNA extraction and PCR}

Genomic DNA of every specimen was extracted by using an E.Z.N.A. Forensic DNA Extraction Kit (D3591-01, Omega Bio-Tek) following the manufacturer's protocol. The primer for internal transcribed spacer (ITS), large ribosomal subunit (LSU), and translation elongation factor (tef1- $\alpha$ ) are ITS4/ITS5, LR5/LROR, and 983f/1567r respectively. The PCR programs are followed in He et al. 2017. The PCR products were sent to commercial company for sequencing, and both directions were sequenced to ensure accuracy.

\section{Phylogenetic analyses}

Details of newly generated and reference sequences retrieved from GenBank were listed in Table 1. Sequences were checked in BioEdit V.7.0.4 first (Hall 2007). Alignments were made by Muscle (Edgar 2004) for each regions separately, including 109 ITS, 82 LSU and 78 tef1- $\alpha$, then adjusted by hand and the ambiguous regions were removed. Final multi-locus matrix comprising 746 bp (base pair) LSU, 545 bp tef1- $\alpha$, and 627 bp ITS. Alignment was submitted to TreeBase (submission ID: 22065). Phylogenetic tree generated by Bayesian Inference (BI) analysis were performed in MrBayes 3.1.2. Two best model for each region were inferred by Mrmodeltest2.2 (Nylander 2004), they are GTR + I + G for ITS and LSU, and SYM + I + G for tef1- $\alpha$. Ten million generations were run for six Markov chains, and sampled every 100th generation resulting in 100,000 trees. Burn-ins was determined in Tracer v1.6 with effective sample sizes (ESS) higher than 200 (http://tree.bio.ed.ac.uk/software/tracer). Remaining trees were used to calculate Bayesian posterior probabilities (PP). Maximum likelihood (ML) analysis and bootstrap values calculation were 
performed in raxmlGUI 1.5b1 with GTRGAMMA model with 1000 replicates (Silvestro \& Michalak 2012). Phylogenetic tree was presented in Fig. 1.

Tabel 1 Taxa information used in the phylogenetic analyses, new taxa are in bold, " $T$ " refers to type

\begin{tabular}{|c|c|c|c|c|}
\hline Species & Collection & ITS & LSU & ef1- $\alpha$ \\
\hline Agaricus aridicola & LAPAG589 & KT951331 & KX084027 & KX198081 \\
\hline A. armandomyces $\mathrm{T}$ & ZRL2015992 & KX684860 & KX684882 & KX684906 \\
\hline A. arrillagarum & LAPAG810 & KF447900 & KX083985 & KT951592 \\
\hline A. badioniveus & LD2012131 & KU975117 & - & - \\
\hline A. boniquamulosus $\mathrm{T}$ & ZRL2010106 & KX657047 & KX656950 & KX684951 \\
\hline A. brunneolus & LAPAG938 & KU975082 & KX083997 & KX198062 \\
\hline A. brunneolutosus $\mathrm{T}$ & MS514 & KU975111 & KX084006 & - \\
\hline A. callacii & LAPAG797 & KF447899 & KX083984 & KX198051 \\
\hline A. campbellensis & GAL9420 & DQ232644 & DQ232657 & - \\
\hline A. campestris $\mathrm{T}$ & LAPAG370 & JQ903618 & KP739803 & KR006636 \\
\hline A. candidolutescens $\mathrm{T}$ & LD2012129 & KT951335 & KT951525 & KT951616 \\
\hline A. catenatus $\mathrm{T}$ & ZRL2012104 & KX657023 & KX656963 & KX684957 \\
\hline A. cerinipileus $\mathrm{T}$ & ZRL2012001 & KX657021 & KX656957 & KX684953 \\
\hline A. cf. kerriganii & WC912 & AY484681 & - & - \\
\hline A. chartaceus & H6271 & JF495048 & - & - \\
\hline A. coccyginus & ZRL2014354 & KU245981 & KX656936 & KX684998 \\
\hline A. colpetei & TL2424 & JX984565 & - & - \\
\hline A. columellatus & MIN938394 & KJ912899 & - & - \\
\hline A. columellatus & SB-2015 & KJ912899 & - & - \\
\hline A. comtulus & LAPAG303 & KU975078 & KX083986 & KX198052 \\
\hline A. dilatostipes & ZRL2014450 & KX656999 & KX656941 & KX685003 \\
\hline A. dulcidulus & PRM909627 & KF447894 & - & KX198064 \\
\hline A. edmondoi & LAPAG412 & KT951326 & KT951481 & KT951590 \\
\hline A. elongatestipes $\mathrm{T}$ & ZRL2013271 & KX657002 & KX656946 & KX684975 \\
\hline A. fimbrimarginatus $\mathrm{T}$ & LD201250 & KU975119 & KX084017 & KX198076 \\
\hline A. flammicolor & LD201225 & KU975115 & KX084010 & KX198070 \\
\hline A. flavopileatus & MS596 & KU975121 & KX084022 & KX198078 \\
\hline A. friesianus & LAPAG592 & KT951316 & KX083992 & KT951594 \\
\hline A. fulvoaurantiacus $\mathrm{T}$ & LD201404 & KU975107 & KX084002 & KX198069 \\
\hline A. gemlii & LAPAG286 & KU975079 & KX083988 & KX198055 \\
\hline A. gemloides $\mathrm{T}$ & ZRL2014084 & KT633271 & KX641405 & KX684986 \\
\hline A. globosporus $\mathrm{T}$ & ZRL2012656 & KX657039 & - & KX684968 \\
\hline A. heinemannianus & LAPAG302 & KF447906 & - & KX198056 \\
\hline A. huijsmanii & LAPAG639 & KF447889 & KT951444 & KT951571 \\
\hline A. jacobi & LAPAG52 & KF447895 & KX083996 & KX198061 \\
\hline A. jingningensis $\mathrm{T}$ & ZRL20151562 & KX684877 & KX684895 & KX684917 \\
\hline A. kerriganii $\mathrm{T}$ & AH44509 & KF447893 & KX083999 & KX198066 \\
\hline A. laeticulus $\mathrm{T}$ & Goossens 5272 & KX671705 & - & - \\
\hline A. lamelliperditus & MDBF61/96 & JX984559 & - & - \\
\hline
\end{tabular}


Table 1 Continued.

\begin{tabular}{|c|c|c|c|c|}
\hline Species & Collection & ITS & LSU & ef1- $\alpha$ \\
\hline A. leucocarpus & LD201226 & KU975102 & KX083982 & KX198049 \\
\hline A. leucocarpus $\mathrm{T}$ & LD201215 & KU975101 & KX084014 & KX198074 \\
\hline A. luteofibrillosus & ZRL2012359 & KU245978 & KX656967 & KX684959 \\
\hline A. luteomaculatus & LAPAG331 & KF447901 & - & KX198053 \\
\hline A. luteopallidus & LD2012113 & KU975124 & KX084026 & KX198080 \\
\hline A. mangaoensis $\mathrm{T}$ & ZRL2010056 & KX657042 & KX656956 & KX684946 \\
\hline A. marisae & LAPAG138 & KU975083 & KX083998 & KX198065 \\
\hline A. matrum $\mathrm{T}$ & LAPAG817 & KF447896 & KX083991 & KX198058 \\
\hline A. megalosporus & ZRL2012199 & KT951367 & KT951470 & KT951595 \\
\hline A. microviolaceus $\mathrm{T}$ & ZRL2012718 & KX657033 & KX656980 & KX684971 \\
\hline A. minipurpureus $\mathrm{T}$ & ZRL2010058 & KX657043 & KX656953 & KX684947 \\
\hline A. neimengguensis $\mathrm{T}$ & ZRL20151845 & KX684870 & KX684902 & KX684924 \\
\hline A. pallens & LAPAG441 & KF447898 & - & KX198067 \\
\hline A. parvibicolor $\mathrm{T}$ & LD2012116 & KP715162 & KX084016 & KX198075 \\
\hline A. parvibrunneus $\mathbf{T}$ & ZRL20161053 & MG137001 & MG196345 & MG196351 \\
\hline A. patris $\mathrm{T}$ & LD201224 & KU975118 & KX084012 & KX198073 \\
\hline A. pseudolutosus & LAPAG454 & КT951329 & KT951453 & КT951602 \\
\hline A. pseudominipurpureus & ZRL2013320 & MG136999 & MG196344 & MG196349 \\
\hline A. pseudominipurpureus $\mathbf{T}$ & ZRL2013341 & MG137000 & MG196343 & MG196350 \\
\hline A. pseudopallens $\mathrm{T}$ & ZRL20151552 & KX684874 & KX684891 & - \\
\hline A. pseudopurpurellus $\mathrm{T}$ & ZRL2014063 & KX656988 & KX641404 & KX684985 \\
\hline A. purpurellus & LAPAG944 & KU975076 & KX083994 & KX198060 \\
\hline A. purpureofibrillosus $\mathrm{T}$ & ZRL3080 & JF691542 & KX084021 & - \\
\hline A. purpureosquameus $\mathrm{T}$ & LE2016047 & KX684878 & - & - \\
\hline A. robustulus & ZRL2012357 & КT951369 & KT951496 & KT951610 \\
\hline A. robustulus $\mathrm{T}$ & CA847 & KU975086 & KX084034 & KX198039 \\
\hline A. rufifibrillosus $\mathrm{T}$ & ZRL20151536 & KX684878 & KX684893 & KX684915 \\
\hline A. rufipileus $\mathrm{T}$ & ZRL2014140 & KX656991 & KX656937 & KX684991 \\
\hline A. sodalis $\mathrm{T}$ & LD2012159 & KP715161 & KX084014 & KX198074 \\
\hline A. stevensii & FS 06-02-09 & KJ877785 & - & - \\
\hline A. viridopurpurascens & Horak68/79 & JF514525 & - & - \\
\hline A. wariatodes & TWM1589 & JF495052 & JF495030 & - \\
\hline A. yanzhiensis & ZRL20162060 & MG137002 & MG196348 & MG196352 \\
\hline A. yanzhiensis & ZRL20162139 & MG137004 & MG196347 & MG196353 \\
\hline A. yanzhiensis $\mathbf{T}$ & ZRL20162082 & MG137003 & MG196346 & - \\
\hline A. sp. & GAL3083 & EF460374 & EF460399 & - \\
\hline A. sp. & ZD1528 & KU975104 & KX083987 & KX198054 \\
\hline A. sp. & ZRL2014380 & KX656998 & KX656932 & KX685000 \\
\hline A. sp. & CA935 & KU975085 & KX084036 & KX198034 \\
\hline A. sp. & MS386 & KU975113 & KX084008 & KX198044 \\
\hline A. sp. & ZRL2010079 & KX657046 & KX656951 & KX684950 \\
\hline A. sp. & ADK3580 & KU975097 & - & - \\
\hline
\end{tabular}


Table 1 Continued.

\begin{tabular}{|c|c|c|c|c|}
\hline Species & Collection & ITS & $\mathbf{L S U}$ & ef1- $\alpha$ \\
\hline A. sp. & NTT72 & JF514539 & - & - \\
\hline A. sp. & PYP014 & KU975091 & - & - \\
\hline A. sp. & CA845 & KU975084 & KX084033 & KX198035 \\
\hline A. sp. & ZRL3056 & JF691541 & KX084020 & - \\
\hline A. sp. & ADK2751 & JF514519 & - & - \\
\hline A. sp. & LD201252 & KU975103 & - & KX198050 \\
\hline A. sp. & ZRL2011039 & KT951351 & KT951449 & KT951606 \\
\hline A. sp. & Vellinga2360 & AF482831 & AF482877 & - \\
\hline A. sp. & TL2154 & JF495059 & - & - \\
\hline A. sp. & TL2307 & JF495058 & - & - \\
\hline A. sp. & ZRL20151437 & KX684876 & KX684892 & KX684914 \\
\hline A. sp. & ZRLWXH3067 & KT951387 & KT951497 & KT951611 \\
\hline A. sp. & CA848 & JF727864 & KT951445 & KT951605 \\
\hline A. sp. & NTS73 & KU975099 & - & - \\
\hline A. sp. & NTT33 & JF514535 & - & - \\
\hline A. sp. & PS036 & KU975087 & KX084035 & KX198036 \\
\hline A. sp. & ZRL20151119 & KX684855 & KX684890 & KX684913 \\
\hline A. sp. & ZRLLD013 & KT951384 & KT951516 & KT951604 \\
\hline A. sp. & ZRLWXH3402 & KX657016 & KX656983 & KX685006 \\
\hline A. sp. & CA843 & JF727866 & KX084029 & KX198040 \\
\hline A. sp. & ZRL2010002 & KX657041 & KX656954 & KY427449 \\
\hline A. sp. & CA846 & JF727865 & KT951452 & KT951601 \\
\hline A. sp. & PDD68575 & AF059224 & AF059224 & - \\
\hline A. sp. & GAL5812 & EF460364 & EF460389 & - \\
\hline A. sp. & MATA774 & JF727871 & - & - \\
\hline A. sp. & LAPAM14 & KT951312 & - & KT951613 \\
\hline A. sp. & ZRLWXH3161 & KT951391 & KT951526 & KT951615 \\
\hline A. sp. & ZRL2012004 & KT951355 & KT951457 & KT951608 \\
\hline
\end{tabular}

\section{Results}

\section{Taxonomy}

Agaricus parvibrunneus M.Q. He, K.D. Hyde \& R.L. Zhao sp. nov.

Fig. 2

Fungal Names: FN570507; Faceoffungi Number: FoF03851

Etymology - The epithet "parvi" ' refers to the small basidiome, "brunneus" refers to the brown fibrils on the cap.

Holotype - China, Beijing, Chaoyang District, Institute of Microbiology, 26 July 2016, collected by Bai Xuming, ZRL20161053 (HMAS 278316 Holotype).

Original description - Pileus $14-27 \mathrm{~mm}$ in diam., convex when young, plane with age, disc subunbonate, margin straight, exceeding lamellae; Surface dry, with brown fibrillose scales against white background, scales triangular, appressed, denser at disc, scattered towards the margin. Context up to $2 \mathrm{~mm}$ thick, flesh, white. Lamellae $2-3 \mathrm{~mm}$ broad, free, crowded, pink first, then brown, edge even, intercalated with lamellulae. Annulus single, membranous, white, pendant, smooth on both 


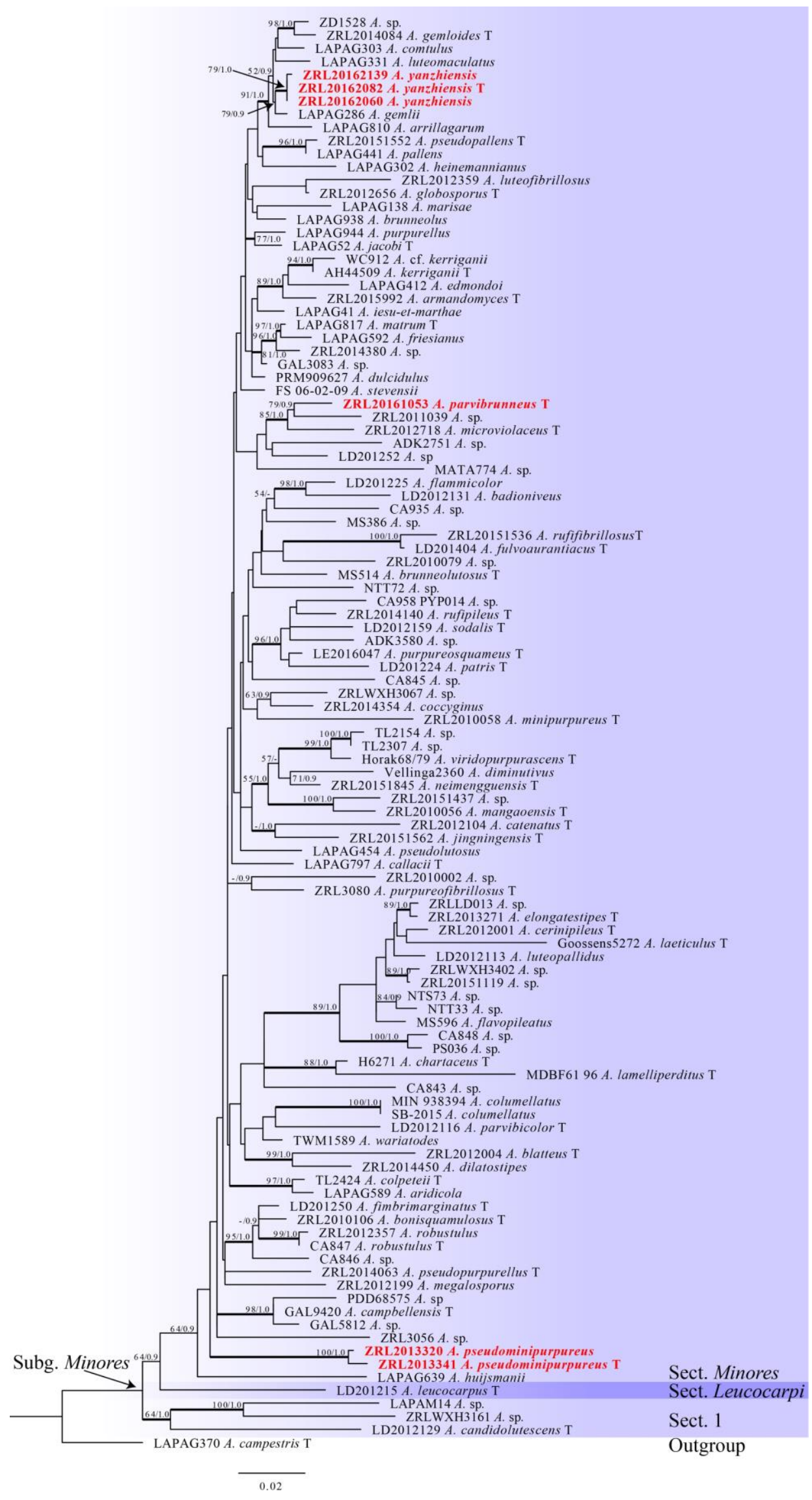

Figure 1 - Maximum likelihood (ML) tree of Agaricus subg. Minores based on LSU, tef1- $\alpha$ and ITS sequences with the outgroup A. campestris L. The bootstrap values and Bayesian posterior probabilities more than 50\%/0.9 (BS/PP) are indicated at the nodes. The branches in bold mean the related $\mathrm{PP}>0.95$, " $\mathrm{T}$ " refers to sequences from type specimen. 
sides. Stipe $21-32 \times 3-7 \mathrm{~mm}$, white, hollow, cylindrical, sometimes with a subbulbous base, surface dry, smooth. Odour of almonds. Basidiome strongly yellow when rubbed.

$\mathrm{KOH}$ reaction - positive yellow. Schäffer's reaction: positive, reddish orange on dry specimen. Basidiospores $5.0-5.8 \times 3.7-4.1 \mu \mathrm{m},\left[\mathrm{x}=5.3 \pm 0.2 \times 3.9 \pm 0.2, \mathrm{Q}=1.3-1.5, \mathrm{Qm}_{\mathrm{m}}=1.4 \pm\right.$ $0.1, \mathrm{n}=20$ ], ellipsoid, smooth, thick-walled, brown. Basidia $13.3-24.7 \times 6.2-7.5 \mu \mathrm{m}$, clavate, hyaline, 4-spored, smooth. Cheilocystidia $14.2-30.0 \times 6.7-15.5 \mu \mathrm{m}$, single, hyaline, smooth, pyriform most, also can be clavate, septa at base, some with yellow pigment inside. Pleurocystidia absent. Pileipellis a cutis composed of hyphae of 5.0-6.6 $\mu \mathrm{m}$ in diam., smooth, cylindrical, hyaline or light brown.

Habitat - solitary on grassland in garden.

Notes - In the phylogenetic analyses, A. parvibrunneus clustered with ZRL2011039/A. sp. and A. microviolaceus M.Q. He \& R.L. Zhao with the BS/PP=79/0.9 value in section Minores. The molecular data shows ZRL2011039 is closely related to A. parvibrunneus. Due to the immature basidiome of ZRL2011039, it was considered as A. sp. in this study. So A. microviolaceus is the only known species phylogenetically close to $A$. parvibrunneus.

Compared in morphology, A. microviolaceus and A. parvibrunneus both have small basidiomes, but $A$. parvibrunneus can be easily distinguished by brown pileus, while A. microviolaceus is purple. Many species in section Minores have small basidiomes (pileus diameter less than $30 \mathrm{~mm}$ ), such as A. blatteus M.Q. He \& R.L. Zhao, A. minipurpureus M.Q. He \& R.L. Zhao, A. purpureofibrillosus Linda J. Chen, R.L. Zhao \& K.D. Hyde, and A. callacii L.A. Parra, R. Iglesias, Fdez. -Vic. \& Oyarzabal. But they all have pinkish and purple fibrils, the pileus color tends to be red while it is brown in A. parvibrunneus. Moreover, A. parvibrunneus has larger basidiospores than those of $A$. blatteus $(4.5 \pm 0.2 \times 3.3 \pm 0.2 \mu \mathrm{m})$ and A. purpureofibrillosus $(4.9 \pm 0.12 \times 2.9 \pm 0.14 \mu \mathrm{m})$, or smaller than those of A. callacii $(6.2 \times 4.9 \mu \mathrm{m})$ (Parra 2013, Chen et al. 2017, He et al. 2017). Agaricus minipurpureus has the same sized basidiospores, but its cheilocystidia are clavate while they are pyriform in A. parvibrunneus. Based on phylogenetic analyses and morphological characteristics, $A$. parvibrunneus is introduced here as a new species, and this new species is characterized by its small basidiomes, brown pileus and pyriform cheilocystidia.

Agaricus pseudominipurpureus M.Q. He, K.D. Hyde \& R.L. Zhao sp. nov.

Fig. 3

Fungal Names: FN570508; Faceoffungi Number: FoF03852

Etymology - refers to the similarity of this new species to A. minipurpureus in morphology.

Holotype - China, Yunnan Province, Dehong County, Tongbiguan natural reserve, $24^{\circ} 61^{\prime} \mathrm{N}$, 9764' E, altitude 1341m, 20 July 2013, collected by Zhao Ruilin, ZRL2013341 (HMAS 278354 Holotype).

Original description - Pileus $14-26 \mathrm{~mm}$ in diam., parabolic first, then convex, finally plane with age, disc unbonate, margin straight, slightly exceeding lamellae, uplifted when mature; Surface dry, with plenty of appressed, purple, reddish brown fibrils against white background, denser at disc, scattered towards the margin. Context up to $2 \mathrm{~mm}$ thick, flesh, white. Lamellae $2-3 \mathrm{~mm}$ broad, free, crowded, pink firstly, then brown, edge even, intercalated with lamellulae. Annulus single, membranous, white, pendant, upper surface smooth, lower surface fibrillose. Stipe $24-43 \times 2-3$ $\mathrm{mm}$, white, hollow, cylindrical, surface dry, surface below the annulus fibrillose. Odour of almonds. Basidiome strongly yellow when rubbed then orange brown immediately.

$\mathrm{KOH}$ reaction - positive yellow. Schäffer's reaction: positive, reddish orange on dry specimen.

Basidiospores $4.3-5 \times 3.1-3.6 \mu \mathrm{m},\left[\mathrm{x}=4.6 \pm 0.2 \times 3.3 \pm 0.1, \mathrm{Q}=1.3-1.5, \mathrm{Qm}_{\mathrm{m}}=1.4 \pm 0.1\right.$, $\mathrm{n}=20$ ], ellipsoid, smooth, thick-walled, brown. Basidia $11-15.2 \times 5-6.6 \mu \mathrm{m}$, clavate, hyaline, 4 spored, smooth. Cheilocystidia absent. Pleurocystidia absent. Pileipellis a cutis composed of hyphae of $6.5-10.5 \mu \mathrm{m}$ in diam., smooth, cylindrical, hyaline or light brown.

Habitat - solitary on soil in forest.

Other specimens examined cChina, Yunnan Province, Dehong County, Tongbiguan natural reserve, $24^{\circ} 61^{\prime} \mathrm{N}, 97^{\circ} 65^{\prime} \mathrm{E}$, altitude $1341 \mathrm{~m}, 20$ July 2013 , collected by Zhao Ruilin, ZRL2013320 (HMAS 278427). 

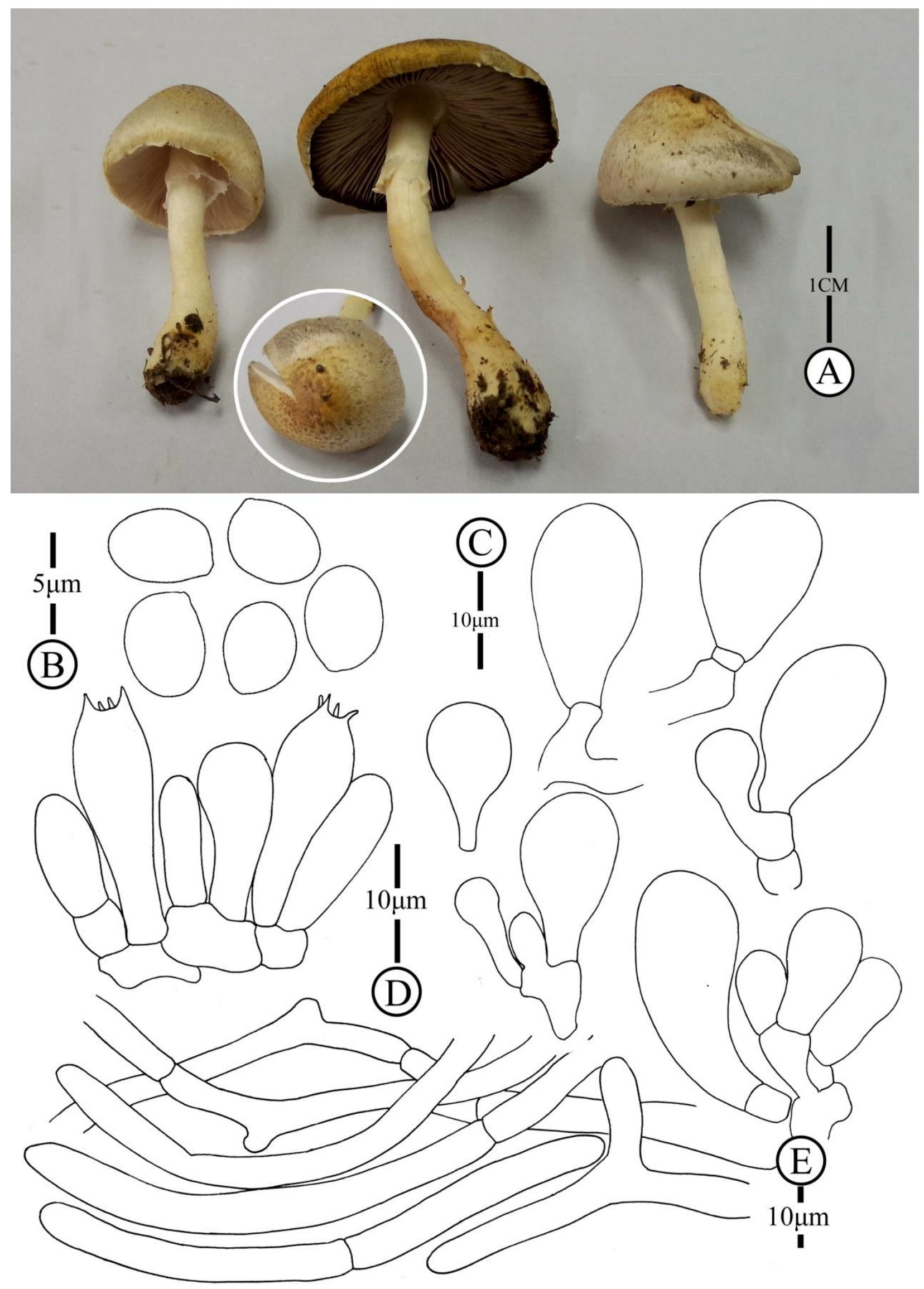

Figure 2 Morphology of Agaricus parvibrunneus, A basidiomes. B basidiospores. C cheilocystidia. $\mathrm{D}$ basidia. E pileipellis hyphae. 
Notes - According to phylogenetic study, the two specimens (ZRL2013320 and ZRL2013341) clustered at the base position of section Minores with fully support (BS/PP $=100 / 1.0$ value), which is representing A. pseudominipurpureus. In the tree A. pseudominipurpureus has a distinct position. But there are many morphologically similar species, such as A. microviolaceus, A. blatteus, A. minipurpureus, A. purpureofibrillosus and A. pseudopurpurellus. They all have small basidiomes (pileus diameter $<30 \mathrm{~mm}$ ), and a pileus covered by purple fibrils (He et al. 2017). In addition, microscopically, the new species differs from all of them by the absence of cheilocystidia. Agaricus pseudopurpurellus is similar to this new species because both have small basidiomes, the same basidiospores in shape and size, and absent cheilocystidia. However, in their ITS sequence, those two species are different at 38 positions. The molecular phylogenetic tree also clearly showed they are different species. Based on phylogenetic and morphological characteristics, A. pseudominipurpureus is introduced here as a new species, and it is characterized by its small basidiomes, absence of cheilocystidia and distinct phylogenetic position in section Minores.

Agaricus yanzhiensis M.Q. He, K.D. Hyde \& R.L. Zhao sp. nov.

Fig. 4

Fungal Names: FN570506; Faceoffungi Number: FoF 03850

Etymology - The epithet "yanzhiensis" refers to the type location, Yanzhi Mountain of Qilianshan National Natural Reserve in China.

Holotype - China, Gansu Province, Shandan County, Yanzhishan Forest Park, $38^{\circ} 78^{\prime} \mathrm{N}$, $101^{\circ} 08^{\prime}$ E, altitude 1765m, 31 August 2016, collected by Dai Rong-chun, ZRL20162082 (HMAS 281083 Holotype).

Original description - Pileus $21-75 \mathrm{~mm}$ in diam., parabolic first, then convex, finally plane with age, disc can be subunbonate when mature, margin straight, also can be uplifted when mature, slightly exceeding lamellae, sometimes with little appendiculate remains of universal veil; Surface dry, covered by appressed, brown or reddish brown fibrils, background white, denser at disc, scattered towards the margin. Context up to $5 \mathrm{~mm}$ thick, flesh, white. Lamellae $5 \mathrm{~mm}$ broad, free, crowded, white first, then pink or reddish brown, brown finally, edge even, intercalated with lamellulae. Annulus single, membranous, white, pendant, upper surface smooth, lower surface fibrillose, sometimes with brown pigment on the edge, connect stipe with white silky fibrils. Stipe $29-65 \times 6$ -8 (12 - 18 at base) $\mathrm{mm}$, white, hollow, cylindrical, some with bulbous base, surface dry, surface below the annulus fibrillose. Odour of almonds. Basidiome strongly yellow when rubbed.

$\mathrm{KOH}$ reaction - positive yellow. Schäffer's reaction: positive, reddish orange on dry specimen. Basidiospores $5.0-5.8 \times 3.7-4.1 \mu \mathrm{m},\left[\mathrm{x}=5.3 \pm 0.2 \times 3.9 \pm 0.2, \mathrm{Q}=1.3-1.5, \mathrm{Qm}_{\mathrm{m}}=1.4 \pm 0.1, \mathrm{n}=\right.$ 20], ellipsoid, smooth, thick-walled, brown. Basidia $13.3-24.7 \times 6.2-7.5 \mu \mathrm{m}$, clavate, hyaline, $4-$ spored, smooth. Cheilocystidia single, smooth, hyaline, pyriform, septa at base. Pleurocystidia absent. Pileipellis a cutis composed of hyphae of $6.5-10.5 \mu \mathrm{m}$ in diam., smooth, cylindrical, hyaline or light brown, slightly constrict at septa.

Habitat - Gregarious on soil in forest.

Other specimens examined - China, Gansu Province, Shandan County, Yanzhishan Forest Park, $38^{\circ} 78^{\prime} \mathrm{N}, 101^{\circ} 08^{\prime} \mathrm{E}$, altitude $1765 \mathrm{~m}, 31$ August 2016, collected by Zhao Rui-lin, ZRL20162060 (HMAS 280994). China, Gansu Province, Sunan County, Dayekou, 38 $83^{\prime}$ N, 99 $61^{\prime}$ E, altitude 2294m, 01 September 2016, collected by Bai Xu-Ming, ZRL20162139 (HMAS 281085).

Notes - Our phylogenetic analyses indicated that A. yanzhiensis is a member of section Minores (Fig. 1). Three specimens (ZRL20162060, ZRL20162082 and ZRL20162139) clustered together representing $A$. yanzhiensis with the statistic support of $\mathrm{BS} / \mathrm{PP}=79 / 1.0$ value, then $A$. yanzhiensis clades with ZD1528/A. sp., A. gemloides, A. comtulus Fr., A. luteomaculatus F.H. Møller and A. gemlii L.A. Parra, Arrillaga, M.Á. Ribes \& Callac with the support of BS/PP=52/0.9 value.

In the morphological study, the phylogenetically closest species is A. gemlii which have six different positions in ITS, one position in LSU and eight positions in tef1- $\alpha$ sequences. Agaricus gemlii can be separated from this new species by its reddish purple fibrils on the pileus while A. yanzhiensis is reddish brown. Furthermore, the habitat of A. gemlii is damp Atlantic wood while this new species is continental cold forest which dominated by Picea crassifolia. There are some more species that 

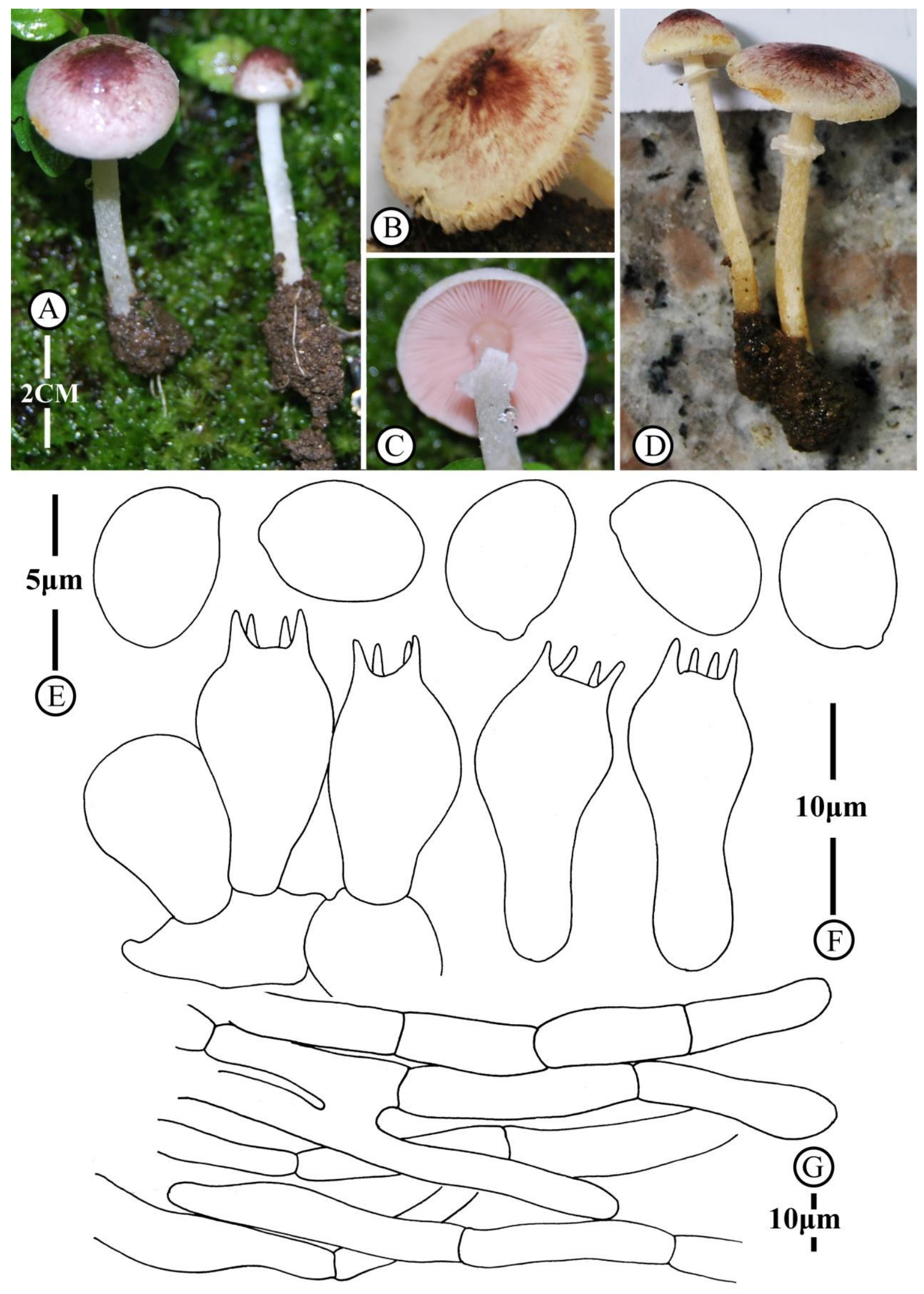

Figure 3 - Morphology of Agaricus pseudominipurpureus, A-D basidiomes in the field. E basidiospores. F basidia. $\mathrm{G}$ pileipellis hyphae. 

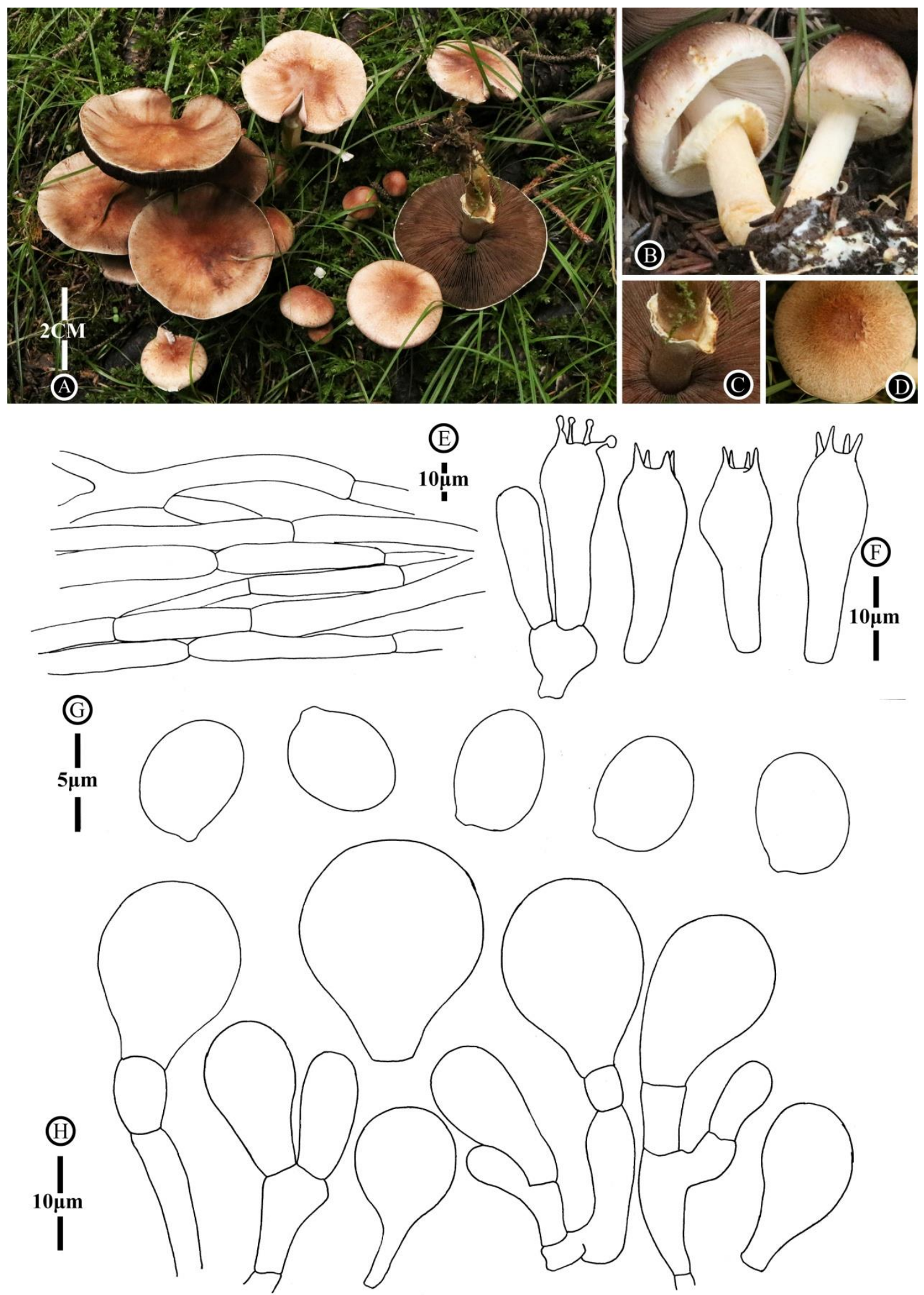

Figure 4 - Morphology of Agaricus yanzhiensis, A-D basidiomes in the field. E pileipellis hyphae. F basidia. G. basidiospores. H Cheilocystidia.

resemble this new species, such as A. comtulus, A. brunneolus (J.E. Lange) Pilát, and A. brunneolutosus Linda J. Chen, Karun. \& K.D. Hyde. In the field they all have middle-sized basidiomes, and brown or reddish brown pileus (Parra 2013), however A. yanzhiensis can be distinguished under the microscope by its larger basidiospores when compared with A. comtulus (4.8 $\times 3.4 \mu \mathrm{m})$ and $A$. brunneolutosus $(4.3 \times 2.9 \mu \mathrm{m})$ (Parra 2013). Agaricus yanzhiensis differs from A. brunneolus by having triangular shaped fibrils scales on the pileus while those of $A$. brunneolus does 
not. In addition, the breadth of lamellae of A. brunneolus (up to $9 \mathrm{~mm}$ ) is nearly double than those of A. yanzhiensis. Based on phylogenetic analyses and morphological characteristics, we introduce $A$. yanzhiensis new to science and this species is characterized by its brown to reddish brown pileus, cheilocystidia pyriform, and often with long narrow stipe, occasionally with one septa at the base.

\section{Discussion}

Members of subgenera Minores and Minoriopsis can be preliminarily separated from other subgenera of Agaricus in the field, because of their relatively small basidiomes, their yellow discoloration when bruised, their simple annulus and their odor of almonds. In addition, species of subgenus Minoriopsis are only known from the Americas. In subgenera Minores, morphological characteristics can be overlapped among species, so the molecular phylogeny analysis is necessary for the identification on species level. Among those three new species we proposed here, $A$. pseudominipurpureus is the example which is hard to distinguish from other known species through morphology, but can be distinguished by molecular data. Presently numerous species of Agaricus section Minores were introduced worldwide. The recent published species in section Minores are A. purpureosquameus and A. rufifibrillosus. Compare with these three new species in this paper morphologically, A. purpureosquameus has small basidiomes, purple pileus and is originally from Thailand. Agaricus rufifibrillosus is originally from east part of China, and it resembles A. yanzhiensis by its medium sized basidiomes, and reddish brown pileus. But under microscope, they can be differed by cheilocystidia which of A. yanzhiensis is pyriform, while A. rufifibrillosus is absent. Up to now, there are 24 species of this section published from China in last three years (He \& Zhao 2015, Li et al. 2016, He et al. 2017, Hyde et al. 2017). Now section Minores has 83 named species including those three new species and 44 of which can be found in China, such as those species which is originally from Thailand: A. patris L.J. Chen, Callac, K.D. Hyde \& R.L. Zhao and A. megalosporus J. Chen, R.L. Zhao, Karunarathna \& K. D. Hyde (Chen et al. 2012, He et al. 2017).

Supplementary note - In the papers "He, M.Q., Chen, J., Zhou, J.L., Cheewangkoon, R., Hyde, K.D. \& Zhao, R.L. (2017). Tropic origins, a dispersal model for saprotrophic mushrooms in Agaricus section Minores with descriptions of sixteen new species, Scientific Reports 7(5122): 1-31", "Hyde, K.D. \& al. (2017). Fungal diversity notes 603-708: taxonomic and phylogenetic notes on genera and species, Fungal Diversity 87:1-235" and "Hyde, K.D., He, M.Q., Zhao, R.L., Perera, R.H., Jayawardena, R.S. \& Camporesi, E. (2017). Nomenclatural novelties. Index Fungorum 347: 1", the authors of six names propose corrections for the epithets of these names, which were incorrectly published due to typographical errors in those papers, as follows:

Agaricus bonussquamulosus M.Q. He \& R.L. Zhao, Sci Rep. 7(5122): 9; Fig. 5. Fungal Names: FN570359 is to be corrected to Agaricus bonisquamulosus M.Q. He \& R.L. Zhao.

Agaricus minorpurpureus M.Q. He \& R.L. Zhao, Sci Rep. 7(5122): 12; Fig. 8. Fungal Names: FN570349 is to be corrected to Agaricus minipurpureus M.Q. He \& R.L. Zhao.

Agaricus cerinupileus M.Q. He \& R.L. Zhao, Sci Rep. 7(5122): 11; Fig. 7. Fungal Names: FN570358 is to be corrected to Agaricus cerinipileus M.Q. He \& R.L. Zhao.

Agaricus rufuspileus M.Q. He \& R.L. Zhao, Sci Rep. 7(5122): 22; Fig. 18. Fungal Names: FN570347 is to be corrected to Agaricus rufipileus M.Q. He \& R.L. Zhao.

Agaricus rufusfibrillosus M.Q. He \& R.L. Zhao, Fungal Divers. 87(1): 188; Fig. 136. Index Fungorum number: IF553825 is to be corrected to Agaricus rufifibrillosus M.Q. He \& R.L. Zhao.

Agaricus purpurlesquameus M.Q. He \& R.L. Zhao, Index Fungorum 347:1. Index Fungorum number: IF552301 is to be corrected to Agaricus purpureosquameus M.Q. He \& R.L. Zhao. Note to 
the readers: This name was previously published in Fungal Divers. 87(1): 185; Fig. 135 but it was invalidly published because two specimens were designated as holotype (Art. 9.1). The name was later validly published in Index Fungorum 347:1.

\section{Acknowledgements}

The National Natural Science Foundation of China (Project ID: 31470152 and 31360014), The Key Lab Supporting Project from Science and Technology Department of Gansu Province (Project ID 145RTSG005); and the Foundation of Innovative Group of Edible Mushrooms Industry of Beijing (Project ID: BAIC05-2017) are thanked for financial supporting.

\section{References}

Chen J, Zhao RL, Karunarathna SC, Callac P et al. 2012 - Agaricus megalosporus: a new species in section Minores. Cryptogamie Mycologie 33, 145-155.

Chen J, Zhao RL, Parra LA, Guelly AK et al. 2015 - Agaricus section Brunneopicti: a phylogenetic reconstruction with descriptions of four new taxa. Phytotaxa. 192, 145-168.

Chen J, Callac P, Parra LA, Karunarathna SC et al. 2017 - Study in Agaricus subgenus Minores and allied clades reveals a new American subgenus and contrasting phylogenetic patterns in Europe and Greater Mekong Subregion. Persoonia 38, 170-196.

Edgar RC 2004 - MUSCLE: multiple sequence alignment with high accuracy and high throughput. Nucleic Acids Research, 32, 1792-1797.

Hall T. 2007 - BioEdit v7. Ibis Biosciences, Carlsbad Available from: http://www.mbio.ncsu.edu/BioEdit/BioEdit.html

He MQ, Zhao RL. 2015 - A new species of Agaricus section Minores from China. Mycology 6, 182186.

He MQ, Chen J, Zhou JL, Cheewangkoon R et al. 2017 - Tropic origins, a dispersal model for saprotrophic mushrooms in Agaricus section Minores with descriptions of sixteen new species. Scientific Reports 7(1): 5122.

Hyde KD, Norphanphoun C, Vanessa PA, Abreu AB et al. 2017 - Fungal diversity notes 603-708: taxonomic and phylogenetic notes on genera and species. Fungal Diversity 87(1): 1-235.

Kerrigan RW. 2016 - Agaricus of North America. New York Botanical Garden Press, Bronx.

Largent DL. 1986 - How to identify mushrooms to genus vol. 1 - 5. Mad River Press, Eureka.

Li GJ, Hyde KD, Zhao RL, S Hongsanan et al. 2016 - Fungal diversity notes 253-366: taxonomic and phylogenetic contributions to fungal taxa. Fungal Diversity 78, 1-237.

Nylander J. 2004 - MrModeltest v2. Program distributed by the author. Evolutionary Biology Centre, Uppsala University.

Parra LA. 2008 - FungiEuropaei, Agaricus L.: Allopsalliota Nauta \& Bas (Parte I), Candusso Edizioni.

Parra LA. 2013 - FungiEuropaei, Agaricus L.: Allopsalliota, Nauta \& Bas (Parte II), Candusso Edizioni.

Silvestro D, Michalak I. 2012 - raxmlGUI: a graphical front-end for RAxML. Org. Divers. Evol. 12, 335-337.

Zhao RL, Karunarathna S, Raspé O, Parra LA et al. 2011 - Major clades in tropical Agaricus. Fungal Diversity 51, 279-296.

Zhao RL, Zhou JL, Chen J, Margaritescu S et al. 2016 - Towards standardizing taxonomic ranks using divergence times-a case study for reconstruction of the Agaricus taxonomic system. Fungal Diversity 78, 239-292. 